DOI: 10.3901/JME.2020.10.200

\title{
铁道车辆物理参数识别方法研究*
}

\author{
陈江雪 周劲松 宫 岛 \\ (同济大学铁道与城市轨道交通研究院 上海 201804)
}

\begin{abstract}
摘要: 针对铁道车辆物理参数识别问题, 首先通过状态空间理论和模态空间理论获得了车辆系统状态矩阵, 接着分别提出了 附加质量法和比例关系法两种铁道车辆物理参数识别方法。附加质量法可识别车辆全部物理参数, 但需要将特定的质量附加 到车辆系统中。比例关系法无需附加质量, 以构架质量参数已知为前提条件来对车辆其余物理参数进行识别。设计了用于物 理参数识别的车辆定向激励工况, 并通过仿真试验对两种物理参数识别方法进行了验证及对比分析。结果表明, 采用附加质 量法进行车辆物理参数识别时, 附加质量大小选取为车辆系统质量的 $3 \%$ 识别精度最高; 附加质量法和比例关系法在识别精 度和测试实施难度方面各有优缺点, 因此, 在实际车辆物理参数识别中, 可根据现场情况, 合理选择识别方法。
\end{abstract}

关键词: 铁道车辆; 参数识别; 状态空间; 模态空间; 物理参数

中图分类号: TG156

\section{Research on Identification Method of Railway Vehicle Physical Parameters}

\author{
CHEN Jiangxue ZHOU Jinsong GONG Dao \\ (Institute of Rail Transit, Tongji University, Shanghai 201804)
}

\begin{abstract}
Aiming at the problem of identifying the physical parameters of railway vehicle, the state matrix of the vehicle system is obtained through state space theory and modal space theory, and then two methods of identifying the physical parameters of railway vehicles are proposed, namely, additional mass method and proportional relationship method. The additional mass method can identify all the physical parameters of the vehicle, but a specific mass needs to be added to the vehicle system. The proportional relationship method does not require additional mass, and the remaining physical parameters of the vehicle are identified on the premise that the frame quality parameter is known. The directional excitation conditions of the vehicle for physical parameter identification are designed, and the two physical parameter identification methods are verified and compared through simulation tests. The results show that adopting the additional mass method, the identification accuracy is highest when the additional mass is selected as $3 \%$ of the vehicle system. The additional mass method and the proportional relationship method have their own advantages and disadvantages in terms of identification accuracy and test implementation difficulty. Therefore, in the actual vehicle physical parameter identification, the identification method needs to be selected according to the site conditions.
\end{abstract}

Key words: railway vehicle; parameter identification; state space; modal space; physical parameter

\section{0 前言}

随着列车运行速度的提高, 对车辆动力学性能 一稳定性、平稳性和曲线通过安全性的要求更高, 而动力学性能主要由车辆的物理参数所决定, 如质 量、惯量、悬挂刚度和悬挂阻尼等参数 ${ }^{[1-5]}$ 。

在车辆研制过程中，质量参数、惯量参数和悬 挂参数等物理参数识别对车辆动力学性能研究有着

* 国家自然科学基金资助项目(51805373)。20190503 收到初稿, 20200306 收到修改稿
十分重要的作用。首先, 可以检验车辆的实际参数 与设计参数是否一致; 其次, 可以考察列车经历一 段里程服役后, 性能退化的悬挂部件参数是否仍然 满足原始设计限界; 最后, 根据识别的参数可以进 行更加精确的理论和仿真计算。因此, 车辆物理参 数识别是理论计算和虚拟样机仿真分析、优化车辆 性能的基本前提。

目前, 针对铁道车辆物理参数的识别研究主要 集中在试验装置和试验方法设计方面。日本铁道机 车车辆工业会在 1986 年制定了在计量轨道上测量 铁道机车车辆的质量、轮重及轴重的测定方法 ${ }^{[6]}$ 。 
日本学者藤田科平等 ${ }^{[7]}$ 采用三点支撑法测定了车体 重心, 并根据车体自由振动周期计算得到了车体转 动惯量。韩国学者 $\mathrm{SONG}$ 等 ${ }^{[8]}$ 提出了一种利用振动 理论对铁路车辆的重心和惯性矩进行测量的方法, 使用四根线悬挂铁路车辆, 通过测量线张力和悬挂 铁路车辆的摆动周期, 分别给出了重心的位置和三 轴向惯性矩。文献[9]依据力系平衡方程和力矩平衡 方程, 采用四支点测力法计算得到了机车重心。文 献[10]结合转向架参数测定试验台, 提出了基于振 动理论的转向架三轴向转动惯量的试验方法, 该方 法必须在安装作动器的试验台上进行测试且需要已 知转向架质量参数。文献[11]基于重量反应理论以 转向架参数测试台为基础开发了基于坡道模拟器的 转向架质心高度测量系统。文献[12]研制的轨道车 辆车体参数测量试验台能够实现对车体的重量、重 心位置及转动惯量等参数的测量。文献[13]对高速 列车转向架悬挂参数的测试方法进行研究, 建立了 转向架悬挂参数的测试模型, 并进行了新型转向架 悬挂参数测试台的初步开发。文献[14]基于二自由 度的车辆模型, 采用灰箱辨识法对二系悬挂参数进 行估计。文献[15-16]基于结构激振试验设备, 设计 了一个车体参数测定专用装置, 可以对车体重量、 重心位置和转动惯量进行测定。该装置需要使用托 梁将车体悬吊起来, 基于力矩平衡原理对车体重心 和重量进行测定, 使用自振法和激振法对车体点头 转动惯量和侧滚转动惯量进行测定, 使用悬吊摆动 法对车体摇头转动惯量进行测定。

综上所述, 以往的车辆物理参数识别方法均需 要专用试验台, 且试验装置结构复杂、占地空间大, 建立整车试验台和进行整车试验的费用较为昂贵, 投入人力物力较多, 安装及试验操作要求高, 而且 无法将车辆质量参数、惯量参数和悬挂参数同时测 得, 因而在车辆开发中受到一定限制。

研究一种简单易行的、可同时辨识铁道车辆质 量参数、惯量参数和悬挂参数的现场识别方法, 具 有十分重要的工程应用价值。因此, 本文基于状态 空间理论和模态空间理论, 分别提出了附加质量法 和比例关系法两种铁道车辆物理参数识别方法, 设 计了用于参数识别的定向激励测试工况, 利用车辆 自由振动加速度响应信号, 对铁道车辆质量参数、 惯量参数和悬挂参数同时识别进行了探索性研究。

\section{1 车辆系统状态矩阵}

由于铁道车辆的垂向与横向动力学存在弱耦合
性 ${ }^{[1,17]}$, 所以往往可以分别进行建模及进行动力学 分析。本文以车辆物理参数中的车体质量、车体点 头惯量、构架质量、构架点头惯量、一系和二系垂 向刚度及阻尼系数参数为识别对象, 因此, 构建如 图 1 所示的车辆垂向动力学模型, 并推导系统的状 态矩阵方程。模型中 $z_{\mathrm{b}}$ 为车体浮沉自由度, $\theta_{\mathrm{b}}$ 为车 体点头自由度, $z_{\mathrm{t}}$ 为构架浮沉自由度, $\theta_{\mathrm{t}}$ 为构架点 头自由度, $z_{\mathrm{w} 1} 、 z_{\mathrm{w} 2} 、 z_{\mathrm{w} 3} 、 z_{\mathrm{w} 4}$ 分别为 1 位 $\sim 4$ 位轮 对受到的垂向激励。 $m_{\mathrm{b}}$ 为车体质量, $J_{\mathrm{b}}$ 为车体点 头转动惯量, $m_{\mathrm{t}}$ 为构架质量, $J_{\mathrm{t}}$ 为构架点头转动惯 量。 $k_{\mathrm{s}} 、 k_{\mathrm{p}}$ 分别为二系垂向刚度和一系垂向刚度, $c_{\mathrm{s}} 、 c_{\mathrm{p}}$ 分别为二系垂向阻尼和一系垂向阻尼。 $l_{\mathrm{b}}$ 为 定距之半, $l_{\mathrm{w}}$ 为轴距之半。

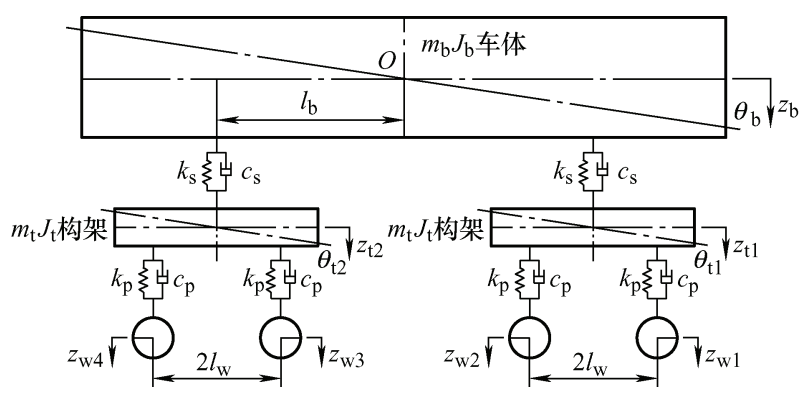

图 1 车辆垂向动力学模型

根据牛顿定律, 车辆系统的动力学方程为

$$
\begin{aligned}
& M \ddot{X}+C \dot{X}+K X=F \\
& \boldsymbol{X}=\left(\begin{array}{llllll}
z_{\mathrm{b}} & \theta_{\mathrm{b}} & z_{\mathrm{t} 1} & \theta_{\mathrm{t} 1} & z_{\mathrm{t} 2} & \theta_{\mathrm{t} 2}
\end{array}\right)^{\mathrm{T}} \\
& \boldsymbol{M}=\operatorname{diag}\left(m_{\mathrm{b}} \quad J_{\mathrm{b}} \quad m_{\mathrm{t}} \quad J_{\mathrm{t}} \quad m_{\mathrm{t}} \quad J_{\mathrm{t}}\right)(3) \\
& \boldsymbol{K}=\left(\begin{array}{cccccc}
2 k_{\mathrm{s}} & 0 & -k_{\mathrm{s}} & 0 & -k_{\mathrm{s}} & 0 \\
0 & 2 k_{\mathrm{s}} l_{\mathrm{b}}^{2} & -k_{\mathrm{s}} l_{\mathrm{b}} & 0 & k_{\mathrm{s}} l_{\mathrm{b}} & 0 \\
-k_{\mathrm{s}} & -k_{\mathrm{s}} l_{\mathrm{b}} & k_{\mathrm{s}}+2 k_{\mathrm{p}} & 0 & 0 & 0 \\
0 & 0 & 0 & 2 k_{\mathrm{p}} l_{\mathrm{w}}^{2} & 0 & 0 \\
-k_{\mathrm{s}} & k_{\mathrm{s}} l_{\mathrm{b}} & 0 & 0 & k_{\mathrm{s}}+2 k_{\mathrm{p}} & 0 \\
0 & 0 & 0 & 0 & 0 & 2 k_{\mathrm{p}} l_{\mathrm{w}}^{2}
\end{array}\right) \\
& \boldsymbol{C}=\left(\begin{array}{cccccc}
2 c_{\mathrm{s}} & 0 & -c_{\mathrm{s}} & 0 & -c_{\mathrm{s}} & 0 \\
0 & 2 c_{\mathrm{s}} l_{\mathrm{b}}^{2} & -c_{\mathrm{s}} l_{\mathrm{b}} & 0 & c_{\mathrm{s}} l_{\mathrm{b}} & 0 \\
-c_{\mathrm{s}} & -c_{\mathrm{s}} l_{\mathrm{b}} & c_{\mathrm{s}}+2 c_{\mathrm{p}} & 0 & 0 & 0 \\
0 & 0 & 0 & 2 c_{\mathrm{p}} l_{\mathrm{w}}^{2} & 0 & 0 \\
-c_{\mathrm{s}} & c_{\mathrm{s}} l_{\mathrm{b}} & 0 & 0 & c_{\mathrm{s}}+2 c_{\mathrm{p}} & 0 \\
0 & 0 & 0 & 0 & 0 & 2 c_{\mathrm{p}} l_{\mathrm{w}}^{2}
\end{array}\right)
\end{aligned}
$$

式(1) (5)中, $\boldsymbol{M} 、 \boldsymbol{C}$ 和 $\boldsymbol{K}$ 分别为车辆系统的 质量矩阵、阻尼矩阵和刚度矩阵; $\boldsymbol{X} 、 \dot{\boldsymbol{X}}$ 和 $\ddot{\boldsymbol{X}}$ 分 别为系统的位移矢量矩阵、速度矢量矩阵和加速度 矢量矩阵; $\boldsymbol{F}$ 是作用在轮对上的激励力输入矩阵, 当车辆系统无外力作用, 由初始状态进行自由振动 时, 式(1)的右端项为 0 , 即, 


$$
M \ddot{X}+C \dot{X}+K \boldsymbol{X}=0
$$

引入状态变量 $\boldsymbol{Y}=\left(\begin{array}{ll}\boldsymbol{X} & \dot{\boldsymbol{X}}\end{array}\right)^{\mathrm{T}}$, 车辆系统的动力学 方程可写成状态方程形式

$$
\dot{\boldsymbol{Y}}=\boldsymbol{A} \boldsymbol{Y}
$$

式中, $\boldsymbol{A}$ 为状态矩阵。

$$
\boldsymbol{A}=\left(\begin{array}{cl}
\boldsymbol{O}_{6 \times 6} & \boldsymbol{I}_{6 \times 6} \\
-\boldsymbol{M}^{-1} \boldsymbol{K} & -\boldsymbol{M}^{-1} \boldsymbol{C}
\end{array}\right)
$$

系统状态矩阵 $\boldsymbol{A}$ 的子矩阵 $\boldsymbol{A}_{21}$ 和 $\boldsymbol{A}_{22}$ 分别为

$$
\begin{aligned}
& A_{21}=-\boldsymbol{M}^{-1} \boldsymbol{K} \\
& \boldsymbol{A}_{22}=-\boldsymbol{M}^{-1} \boldsymbol{C}
\end{aligned}
$$

由于车辆系统状态矩阵中包含车辆物理参数, 所以识别车辆系统的状态矩阵是获得车辆物理参数 的前提。

\section{2 系统状态矩阵识别理论}

状态空间理论 ${ }^{[18]}$ 和模态空间理论 ${ }^{[19]}$ 是识别系 统状态矩阵的两类方法, 前者是基于物理坐标系下 的时域参数识别方法, 后者是基于模态坐标系下的 时域参数识别方法。本节将分别对两种方法进行详 细阐述。

\section{1 状态空间理论}

在离散时域, 状态空间方程式(7)可以表示为

$$
\begin{gathered}
\overline{\boldsymbol{Z}}=\boldsymbol{G} \boldsymbol{Z} \\
\boldsymbol{Z}=\left(\begin{array}{llll}
\boldsymbol{Y}(1) & \boldsymbol{Y}(2) & \cdots & \boldsymbol{Y}(N)
\end{array}\right) \\
\overline{\boldsymbol{Z}}=\left(\begin{array}{llll}
\boldsymbol{Y}(2) & \boldsymbol{Y}(3) & \cdots & \boldsymbol{Y}(N+1)
\end{array}\right) \\
\boldsymbol{Y}(k)=\left(\begin{array}{lll}
\boldsymbol{X}(k) & \boldsymbol{X}(k+1)
\end{array}\right)^{\mathrm{T}}
\end{gathered}
$$

式中, $\boldsymbol{Y}(k)$ 为测量的离散矢量; $N$ 为离散矢量的采 样点个数; $\boldsymbol{X}(k)$ 表示传感器在 $t=k \Delta t$ 时刻的测量 值。传递矩阵可以用最小二乘法确定

$$
\boldsymbol{G}=\overline{\boldsymbol{Z}} \boldsymbol{Z}^{\mathrm{T}}\left(\boldsymbol{Z} \boldsymbol{Z}^{\mathrm{T}}\right)^{-1}
$$

另一方面, 根据连续系统与离散系统的关系, 传递矩阵可以表示为

$$
\boldsymbol{G}=\exp (\boldsymbol{A} \Delta t)
$$

式中, $\Delta t$ 为采样间隔。

由式(15) 可以知道系统传递矩阵 $\boldsymbol{G}$ 的特征值 $s_{i}$ 和特征矢量 $\Phi_{i}$ 与系统状态矩阵 $\boldsymbol{A}$ 的特征值 $\lambda_{i}$ 和特 征矢量 $p_{i}$ 存在以下关系

$$
\left\{\begin{array}{l}
s_{i}=\exp \left(\lambda_{i} \Delta t\right) \\
\Phi_{i}=p_{i}
\end{array} \quad i=1,2, \cdots, 2 n\right.
$$

根据式(17)可通过系统传递矩阵的特征值和特 征矢量求得系统状态矩阵的特征值和特征矢量。然 后由矩阵的谱分解理论 ${ }^{[20]}$ 可知, 系统状态矩阵 $\boldsymbol{A}$ 与 其特征值矩阵 $\boldsymbol{\Lambda}$ 、特征矢量矩阵 $\boldsymbol{P}$ 存在以下关系

$$
\boldsymbol{A}=\boldsymbol{P} \boldsymbol{\Lambda} \boldsymbol{P}^{-1}
$$

$$
\begin{gathered}
\boldsymbol{\Lambda}=\operatorname{diag}\left(\begin{array}{llll}
\lambda_{1} & \lambda_{2} & \cdots & \lambda_{n}
\end{array}\right) \\
\boldsymbol{P}=\left(\begin{array}{llll}
p_{1} & p_{2} & \cdots & p_{n}
\end{array}\right)
\end{gathered}
$$

通过式(18)可以重构系统状态矩阵。

\section{2 模态空间理论}

IBRAHIM $^{[21]}$ 提出一种基于模态空间理论的时 域参数识别方法。基于识别的模态参数, 可以重构 系统状态矩阵。对于车辆的动力学方程(1), 由振 动方程的复模态理论可知, 一个 $n$ 自由度的有阻 尼系统含有 $n$ 对复共轭的特征值, 这 $n$ 对复共轭 的特征值与系统的 $n$ 个模态对应, 振动方程的解 可以表示成 $n$ 个模态的解的叠加。微分方程的解 可以表示为

$\left(\begin{array}{c}x_{1}(t) \\ x_{2}(t) \\ \vdots \\ x_{n}(t)\end{array}\right)=\left(\begin{array}{cccc}\Phi_{11} & \Phi_{12} & \cdots & \Phi_{1(2 n)} \\ \Phi_{21} & \Phi_{22} & \cdots & \Phi_{2(2 n)} \\ \vdots & \vdots & & \vdots \\ \Phi_{n 1} & \Phi_{n 2} & \cdots & \Phi_{n(2 n)}\end{array}\right) \cdot\left(\begin{array}{c}\exp \left(\lambda_{1} t\right) \\ \exp \left(\lambda_{2} t\right) \\ \vdots \\ \exp \left(\lambda_{2 n} t\right)\end{array}\right)$

式中, $x_{i}(t)$ 表示系统第 $i$ 个自由度的位移响应, $\lambda_{i}$ 表 示系统的第 $i$ 个特征值, $\Phi_{i j}$ 表示第 $j$ 个特征值对应 第 $i$ 个自由度的振型系数。

为了求解系统的 $n$ 对复共轭特征值, 构造以下 信号矩阵

$$
\begin{aligned}
& \left(\begin{array}{cccc}
x_{1}\left(t_{1}\right) & x_{1}\left(t_{2}\right) & \cdots & x_{1}\left(t_{m}\right) \\
x_{2}\left(t_{1}\right) & x_{2}\left(t_{2}\right) & \cdots & x_{2}\left(t_{m}\right) \\
\vdots & \vdots & & \vdots \\
x_{2 n}\left(t_{1}\right) & x_{2 n}\left(t_{2}\right) & \cdots & x_{2 n}\left(t_{m}\right)
\end{array}\right)= \\
& \left(\begin{array}{cccc}
\Phi_{11} & \Phi_{12} & \cdots & \Phi_{1(2 n)} \\
\Phi_{21} & \Phi_{22} & \cdots & \Phi_{2(2 n)} \\
\vdots & \vdots & & \vdots \\
\Phi_{(2 n) 1} & \Phi_{(2 n) 2} & \cdots & \Phi_{(2 n)(2 n)}
\end{array}\right) .
\end{aligned}
$$

$$
\left(\begin{array}{cccc}
\exp \left(\lambda_{1} t_{1}\right) & \exp \left(\lambda_{1} t_{2}\right) & \cdots & \exp \left(\lambda_{1} t_{m}\right) \\
\exp \left(\lambda_{2} t_{1}\right) & \exp \left(\lambda_{2} t_{2}\right) & \cdots & \exp \left(\lambda_{2} t_{m}\right) \\
\vdots & \vdots & & \vdots \\
\exp \left(\lambda_{2 n} t_{1}\right) & \exp \left(\lambda_{2 n} t_{2}\right) & \cdots & \exp \left(\lambda_{2 n} t_{m}\right)
\end{array}\right)
$$

上述方程可以用矩阵形式表示为

$$
\boldsymbol{Z}_{2 n \times m}=\boldsymbol{\Phi}_{2 n \times 2 n} \boldsymbol{\Gamma}_{2 n \times m}
$$

将式(22)表示的信号矩阵延时 $\Delta t$ 后为 


$$
\left(\begin{array}{cccc}
x_{1}\left(t_{1}+\Delta t\right) & x_{1}\left(t_{2}+\Delta t\right) & \cdots & x_{1}\left(t_{m}+\Delta t\right) \\
x_{2}\left(t_{1}+\Delta t\right) & x_{2}\left(t_{2}+\Delta t\right) & \cdots & x_{2}\left(t_{m}+\Delta t\right) \\
\vdots & \vdots & & \vdots \\
x_{2 n}\left(t_{1}+\Delta t\right) & x_{2 n}\left(t_{2}+\Delta t\right) & \cdots & x_{2 n}\left(t_{m}+\Delta t\right)
\end{array}\right)=\left(\begin{array}{cccc}
\bar{\Phi}_{11} & \bar{\Phi}_{12} & \cdots & \bar{\Phi}_{1(2 n)} \\
\bar{\Phi}_{21} & \bar{\Phi}_{22} & \cdots & \bar{\Phi}_{2(2 n)} \\
\vdots & \vdots & & \vdots \\
\bar{\Phi}_{(2 n) 1} & \bar{\Phi}_{(2 n) 2} & \cdots & \bar{\Phi}_{(2 n)(2 n)}
\end{array}\right) \cdot\left(\begin{array}{cccc}
\exp \left(s_{1} t_{1}\right) & \exp \left(s_{1} t_{2}\right) & \cdots & \exp \left(s_{1} t_{m}\right) \\
\exp \left(s_{2} t_{1}\right) & \exp \left(s_{2} t_{2}\right) & \cdots & \exp \left(s_{2} t_{m}\right) \\
\vdots & \vdots & \vdots \\
\exp \left(s_{2 n} t_{1}\right) & \exp \left(s_{2 n} t_{2}\right) & \cdots & \exp \left(s_{2 n} t_{m}\right)
\end{array}\right)
$$

用矩阵形式表示为

$$
\overline{\boldsymbol{Z}}_{2 n \times m}=\overline{\boldsymbol{\Phi}}_{2 n \times 2 n} \boldsymbol{\Gamma}_{2 n \times m}
$$

通过比较式(23)和式(25)可知, 满足以下关系

$$
\overline{\boldsymbol{Z}}_{2 n \times m}=\boldsymbol{G}_{2 n \times 2 n} \boldsymbol{Z}_{2 n \times m}
$$

式中, $\boldsymbol{G}$ 为系统的传递矩阵, 且满足以下关系

$$
\boldsymbol{G}_{2 n \times 2 n}=\boldsymbol{\Phi}_{2 n \times 2 n} \operatorname{diag}
$$

$\left(\begin{array}{llll}\exp \left(\lambda_{1} \Delta t\right) & \exp \left(\lambda_{2} \Delta t\right) & \cdots & \left.\exp \left(\lambda_{2 n} \Delta t\right)\right) \Phi^{-1}{ }_{2 n \times m}\end{array}\right.$

由式(27)可以知道系统传递矩阵 $\boldsymbol{G}$ 的特征值 $s_{i}$ 和特征矢量 $\Phi_{i}$ 与系统状态矩阵 $\boldsymbol{A}$ 的特征值 $\lambda_{i}$ 和特 征矢量 $p_{i}$ 存在以下关系

$$
\left\{\begin{array}{l}
s_{i}=\exp \left(\lambda_{i} \Delta t\right) \quad i=1,2, \cdots, 2 n \\
\Phi_{i}=p_{i}
\end{array}\right.
$$

根据式(28)可求得系统状态矩阵的特征值和特征 矢量。然后系统状态矩阵 $\boldsymbol{A}$ 可以通过式(18) (20)求得。

\section{3 系统物理参数识别方法}

本节基于通过状态空间理论和模态空间理论获 得的车辆系统状态矩阵, 提出附加质量法和比例关系 法来识别车辆物理参数。其中, 附加质量法可识别车 辆全部物理参数, 但需将特定的质量附加到车辆系统 中, 而比例关系法以构架质量参数已知为前提条件来 对车辆其余物理参数进行识别, 但无需附加质量。

\section{1 附加质量法}

根据式(7)所示的系统状态矩阵, 为获得质量矩 阵 $\boldsymbol{M}$ 、阻尼矩阵 $\boldsymbol{C}$ 和刚度矩阵 $\boldsymbol{K}$, 可将式(29)所示 的特定质量矩阵 $\Delta \boldsymbol{M}$ 附加到系统状态矩阵中。

$$
A_{21}=\left(\begin{array}{ccc}
\frac{-2 k_{\mathrm{s}}}{m_{\mathrm{b}}} & 0 & \frac{k_{\mathrm{s}}}{m_{\mathrm{b}}} \\
0 & \frac{-2 k_{\mathrm{s}} l_{\mathrm{b}}^{2}}{J_{\mathrm{b}}} & \frac{l_{\mathrm{b}} k_{\mathrm{s}}}{J_{\mathrm{b}}} \\
\frac{k_{\mathrm{s}}}{m_{\mathrm{t}}} & \frac{l_{\mathrm{b}} k_{\mathrm{s}}}{m_{\mathrm{t}}} & \frac{-2 k_{\mathrm{p}}-k_{\mathrm{s}}}{m_{\mathrm{t}}} \\
0 & 0 & 0 \\
\frac{k_{\mathrm{s}}}{m_{\mathrm{t}}} & \frac{-l_{\mathrm{b}} k_{\mathrm{s}}}{m_{\mathrm{t}}} & 0 \\
0 & 0 & 0
\end{array}\right.
$$

$\Delta \boldsymbol{M}=\operatorname{diag}\left(\begin{array}{llllll}\Delta m_{\mathrm{b}} & \Delta J_{\mathrm{b}} & \Delta m_{\mathrm{t}} & \Delta J_{\mathrm{t}} & \Delta m_{\mathrm{t}} & \left.\Delta J_{\mathrm{t}}\right)\end{array}\right.$

式中, $\Delta m_{\mathrm{b}} 、 \Delta J_{\mathrm{b}} 、 \Delta m_{\mathrm{t}} 、 \Delta J_{\mathrm{t}}$ 分别表示车体附加的质 量、车体附加的点头转动惯量、构架附加的质量、 构架附加的点头转动惯量。则附加质量后的系统状 态矩阵 $\overline{\boldsymbol{A}}$ 为

$$
\overline{\boldsymbol{A}}=\left(\begin{array}{cc}
\boldsymbol{O}_{6 \times 6} & \boldsymbol{I}_{6 \times 6} \\
-(\boldsymbol{M}+\Delta \boldsymbol{M})^{-1} \boldsymbol{K} & -(\boldsymbol{M}+\Delta \boldsymbol{M})^{-1} \boldsymbol{C}
\end{array}\right)
$$

此时, 系统状态矩阵 $\bar{A}$ 的子矩阵 $\overline{\boldsymbol{A}}_{21}$ 和 $\overline{\boldsymbol{A}}_{22}$ 分 别为

$$
\begin{aligned}
& \overline{\boldsymbol{A}}_{21}=-(\boldsymbol{M}+\Delta \boldsymbol{M})^{-1} \boldsymbol{K} \\
& \overline{\boldsymbol{A}}_{22}=-(\boldsymbol{M}+\Delta \boldsymbol{M})^{-1} \boldsymbol{C}
\end{aligned}
$$

对比式(8)和式(9), 可得

$$
\boldsymbol{M}=\Delta \boldsymbol{M} \overline{\boldsymbol{A}}_{21}\left(\boldsymbol{A}_{21}-\overline{\boldsymbol{A}}_{21}\right)^{-1}
$$

或

$$
M=\Delta M \bar{A}_{22}\left(A_{22}-\bar{A}_{22}\right)^{-1}
$$

依据式(33)或式(34)可求得系统的质量矩阵 $\boldsymbol{M}$, 随后, 通过求解式(31)可得系统刚度矩阵 $\boldsymbol{K}$, 同样, 通过求解式(32)可得系统阻尼矩阵 $\boldsymbol{C}$ 。最后, 应用最小二乘法由识别的质量矩阵、阻尼矩阵、刚 度矩阵计算得到系统相应的物理参数。

值得注意的是, 根据式(33)或式(34)可知, 附加 质量矩阵 $\Delta \boldsymbol{M}$ 的选取会直接影响附加质量法的参数 识别结果。

\section{2 比例关系法}

将式(3) (5)的矩阵 $\boldsymbol{M} 、 \boldsymbol{C}$ 和 $\boldsymbol{K}$ 分别代入系统 状态矩阵 $\boldsymbol{A}$ 的子矩阵式(8)和式(9)中, 可得

$$
\left.\begin{array}{ccc}
0 & \frac{k_{\mathrm{s}}}{m_{\mathrm{b}}} & 0 \\
0 & \frac{-l_{\mathrm{b}} k_{\mathrm{s}}}{J_{\mathrm{b}}} & 0 \\
0 & 0 & 0 \\
\frac{-2 l_{\mathrm{w}}^{2} k_{\mathrm{p}}}{J_{\mathrm{t}}} & 0 & 0 \\
0 & \frac{-2 k_{\mathrm{p}}-k_{\mathrm{s}}}{m_{\mathrm{t}}} & 0 \\
0 & 0 & \frac{-2 l_{\mathrm{w}}^{2} k_{\mathrm{p}}}{J_{\mathrm{t}}}
\end{array}\right)
$$




$$
A_{22}=\left(\begin{array}{cccccc}
\frac{-2 c_{\mathrm{s}}}{m_{\mathrm{b}}} & 0 & \frac{c_{\mathrm{s}}}{m_{\mathrm{b}}} & 0 & \frac{c_{\mathrm{s}}}{m_{\mathrm{b}}} & 0 \\
0 & \frac{-2 c_{\mathrm{s}} l_{\mathrm{b}}^{2}}{J_{\mathrm{b}}} & \frac{l_{\mathrm{b}} c_{\mathrm{s}}}{J_{\mathrm{b}}} & 0 & \frac{-l_{\mathrm{b}} c_{\mathrm{s}}}{J_{\mathrm{b}}} & 0 \\
\frac{c_{\mathrm{s}}}{m_{\mathrm{t}}} & \frac{l_{\mathrm{b}} c_{\mathrm{s}}}{m_{\mathrm{t}}} & \frac{-2 c_{\mathrm{p}}-c_{\mathrm{s}}}{m_{\mathrm{t}}} & 0 & 0 & 0 \\
0 & 0 & 0 & \frac{-2 l_{\mathrm{w}}^{2} c_{\mathrm{p}}}{J_{\mathrm{t}}} & 0 & 0 \\
\frac{c_{\mathrm{s}}}{m_{\mathrm{t}}} & \frac{-l_{\mathrm{b}} c_{\mathrm{s}}}{m_{\mathrm{t}}} & 0 & 0 & \frac{-2 c_{\mathrm{p}}-c_{\mathrm{s}}}{m_{\mathrm{t}}} & 0 \\
0 & 0 & 0 & 0 & 0 & \frac{-2 l_{w}^{2} c_{\mathrm{p}}}{J_{\mathrm{t}}}
\end{array}\right)
$$

通过观察矩阵中对角元素之间的比例关系可以 得到

$$
\left\{\begin{array}{l}
m_{\mathrm{b}}=\left(\boldsymbol{A}_{21}(3,1)\right) \times\left(\boldsymbol{A}_{21}(1,3)\right)^{-1} \times m_{\mathrm{t}} \\
J_{\mathrm{b}}=\left(\boldsymbol{A}_{21}(3,2)\right) \times\left(\boldsymbol{A}_{21}(2,3)\right)^{-1} \times m_{\mathrm{t}} \\
k_{\mathrm{s}}=\left(\boldsymbol{A}_{21}(3,1)\right) \times m_{\mathrm{t}} \\
c_{\mathrm{s}}=\left(\boldsymbol{A}_{22}(3,1)\right) \times m_{\mathrm{t}} \\
k_{\mathrm{p}}=-\left(\boldsymbol{A}_{21}(3,3)+\boldsymbol{A}_{21}(3,1)\right) \times m_{\mathrm{t}} / 2 \\
c_{\mathrm{p}}=-\left(\boldsymbol{A}_{22}(3,3)+\boldsymbol{A}_{22}(3,1)\right) \times m_{\mathrm{t}} / 2 \\
J_{\mathrm{t}}=2 l_{\mathrm{w}}^{2}\left(\boldsymbol{A}_{21}(3,3)+\boldsymbol{A}_{21}(3,1)\right) \times m_{\mathrm{t}} / 2 \boldsymbol{A}_{21}(4,4)
\end{array}\right.
$$

由式(37)可知, 根据矩阵 $\boldsymbol{A}$ 的子矩阵中对角元 素之间的比例关系, 在已知构架质量的前提下, 可 以通过比例关系法直接计算得到车体的质量参数和 惯量参数、构架的惯量参数以及车辆的悬挂参数。

\section{4 物理参数识别方法验证及对比分析}

为了验证上述车辆参数识别方法的有效性, 针 对第 1 节所述车辆系统动力学方程, 基于表 1 所示 的某型高速列车参数, 采用 Matlab/simulink 仿真平 台搭建车辆模型, 基于状态空间理论和模态空间理 论进行铁道车辆参数识别。

表 1 某型高速列车参数及其含义

\begin{tabular}{cc}
\hline 物理量 & 数值 \\
\hline 车体质量 $/ \mathrm{kg}$ & 36000 \\
车体点头惯量 $/\left(\mathrm{kg} \cdot \mathrm{m}^{2}\right)$ & 2300000 \\
构架质量 $/ \mathrm{kg}$ & 2100 \\
构架点头惯量 $/\left(\mathrm{kg} \cdot \mathrm{m}^{2}\right)$ & 2100 \\
二系垂向刚度 $/(\mathrm{N} / \mathrm{m})$ & 260000 \\
二系垂向阻尼 $/ \mathrm{N} \cdot \mathrm{s} / \mathrm{m})$ & 20000 \\
一系垂向刚度 $/(\mathrm{N} / \mathrm{m})$ & 600000 \\
一系垂向阻尼 $/(\mathrm{N} \cdot \mathrm{s} / \mathrm{m})$ & 30000 \\
定距之半 $/ \mathrm{m}$ & 9 \\
轴距之半 $/ \mathrm{m}$ & 1.25 \\
\hline
\end{tabular}

\section{1 参数识别方法激励工况设计}

根据式(15)和式(26)可知, 在参数识别过程中, 为构造系统传递函数矩阵, 首先需要分别获得车体 浮沉响应信号、车体点头响应信号、构架浮沉响应 信号及构架点头响应信号。为此, 设计如下三种定 向激励测试工况: (1) 车体浮沉和构架浮沉模态激励 (令车辆八个车轮同时通过同一高度的楔块后垂直 落地, 模拟阶跃激励输入, 如图 2 所示); (2) 车体 点头模态激励(令车辆前转向架(或后转向架)四个车 轮同时通过同一高度的楔块后垂直落地，模拟阶跃 激励输入，如图 3 所示); (3) 构架点头模态激励(令 车辆前转向架前轮对(或后轮对)或后转向架前轮对 (或后轮对)通过同一高度的楔块后垂直落地，模拟 阶跃激励输入，如图 4 所示)。上述三种定向激励测 试工况不受专用的车辆参数识别试验装置所限, 易 于在车辆存放现场实施。本文通过仿真模型分别模 拟实现上述三种定向激励。

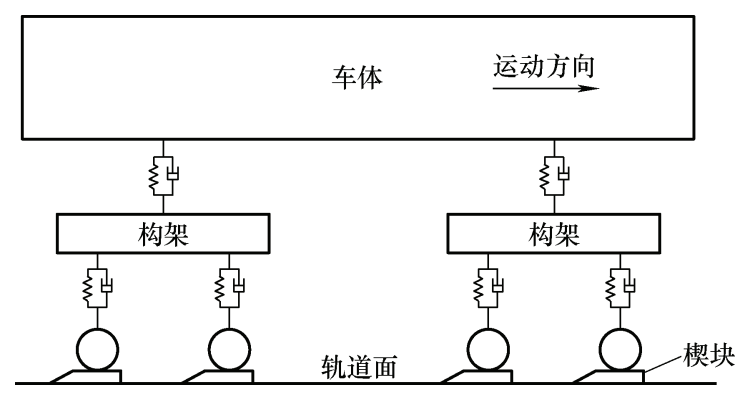

图 2 车体浮沉和构架浮沉模态激励

\section{2 附加质量优化选取}

由于采用附加质量法进行参数识别时, 附加质 量的大小可能会对识别结果的精度产生影响, 且如 果附加质量过大, 会增加测试成本及难度, 因此合 理选择附加质量大小十分必要。

对所建立的车辆模型施加第 4.1 节所述的三种 定向激励, 模拟阶跃激励所使用的楔块高度为 


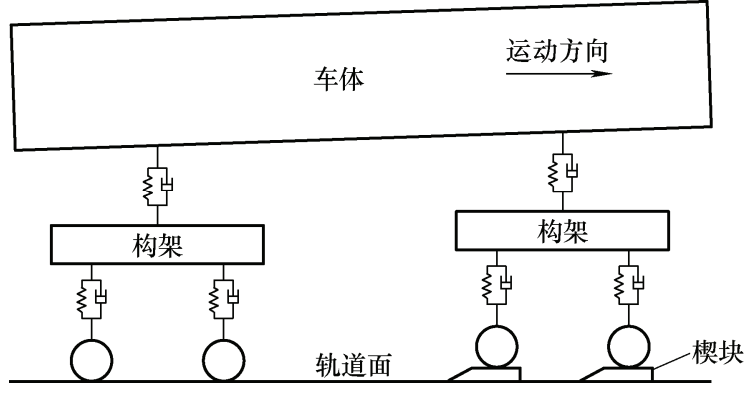

图 3 车体点头模态激励

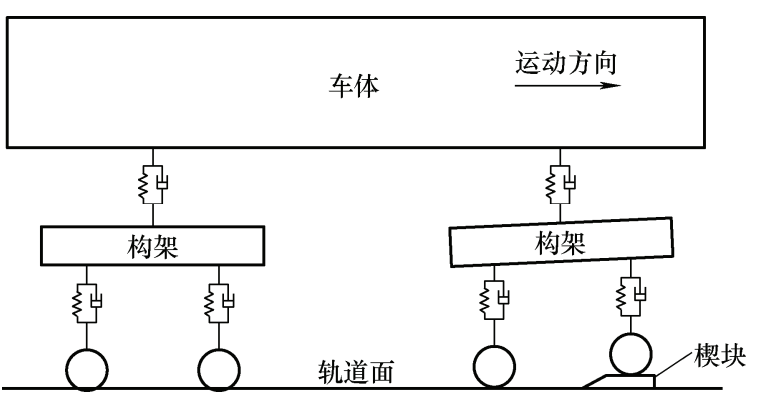

图 4 构架点头模态激励

$16 \mathrm{~mm}$, 依次获得车体浮沉响应信号、车体点头响 应信号、构架浮沉响应信号及构架点头响应信号, 然后, 分别采用基于状态空间理论的附加质量法和 基于模态空间理论的附加质量法对车辆系统物理参 数进行识别。图 5 所示为采用基于状态空间理论的 附加质量法时, 附加质量大小对识别参数误差的影 响。其中, 附加质量 $\Delta \boldsymbol{M}$ 以车辆系统质量 $\boldsymbol{M}$ 的百分 比为参考, 选取范围为 $1 \%$ 至 $30 \%$ 。从图 5 中可以 看到, 当附加质量小于系统质量的 $3 \%$ 时, 随着附加 质量的增大, 全部车辆参数识别误差均在较大范围 内波动; 当附加质量为系统质量的 $3 \%$ 时, 全部车辆 参数识别误差均达到最小值; 当附加质量大于系统 质量的 3\%时, 随着附加质量的增大, 车体点头转动 惯量的识别误差保持在 $-0.05 \%$ 附近波动, 而其他参 数的识别误差则逐渐收敛至较小范围内波动。

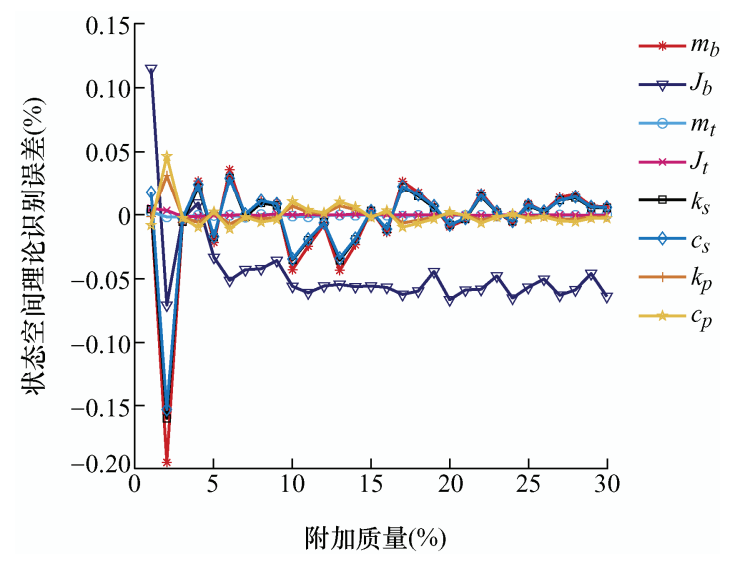

图 5 附加质量对基于状态空间理论的 附加质量法识别误差的影响
图 6 所示为采用基于模态空间理论的附加质量 法时, 附加质量大小对识别参数误差的影响。其中, 附加质量大小选取范围为系统质量的 1\%至 30\%。 从图 6 中可以看到, 随着附加质量的增大, 车体点 头转动惯量的识别误差先增大后逐渐减小, 而其他 参数的识别误差则是逐渐收玫至较小值, 当附加质 量为系统质量的 3\%时, 总体车辆参数识别误差达到 较小值。

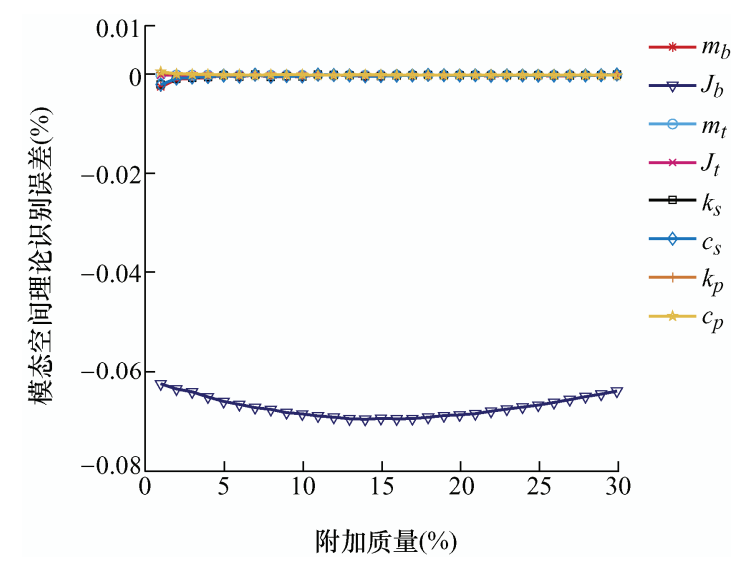

图 6 附加质量对基于模态空间理论的 附加质量法识别误差的影响

综上所述，基于状态空间理论和基于模态空间 理论的附加质量法对车辆系统物理参数的识别误差 均较小, 因此, 采用附加质量法进行车辆系统物理 参数识别是有效的, 且附加质量大小选取为车辆系 统质量的 3\%识别精度最高。

\section{3 识别方法对比分析}

本节将对附加质量法和比例关系法两种铁道车 辆物理参数识别方法进行对比分析。其中, 在采用 附加质量法进行参数识别时, 附加质量大小选取为 车辆系统质量的 $3 \%$ 。

表 2 所示为基于状态空间理论的附加质量法和 比例关系法的识别结果及相应误差。表 3 所示为基 于模态空间理论的附加质量法和比例关系法的识别 结果及相应误差。对比表 2 和表 3 的识别结果, 可 以得出以下结论。

(1) 两种物理参数识别方法的识别误差均在可 接受范围内, 表明这两种方法是有效的。

（2）就车体质量参数识别而言，基于状态空间 理论的附加质量法绝对误差为 $0.0558 \%$, 而比例关 系法绝对误差为 $0.0009 \%$; 基于模态空间理论的附 加质量法绝对误差为 0.006 5\%, 而比例关系法绝对 误差为 $0.0001 \%$ 。可见关于车体质量参数识别, 基 于状态空间理论的识别精度要低于模态空间理论, 附加质量法的识别精度要低于比例关系法。 
(3) 就车体点头转动惯量参数识别而言, 基于 状态空间理论的附加质量法绝对误差为 $0.0268 \%$, 而比例关系法绝对误差为 6.396 5\% ; 基于模态空间 理论的附加质量法绝对误差为 $0.6424 \%$, 而比例关 系法绝对误差为 $6.2565 \%$ 。可见关于车体点头转动 惯量参数识别, 附加质量法的识别精度要高于比例 关系法, 且基于状态空间理论的附加质量法识别精 度要高于基于模态空间理论的附加质量法。

（4）附加质量法可以对构架质量参数进行识 别, 基于状态空间理论的识别精度要低于模态空间 理论。

(5) 关于构架点头转动惯量参数和悬挂参数识 别, 基于状态空间理论的识别精度低于模态空间理 论, 附加质量法的识别精度低于比例关系法。

表 2 状态空间理论物理参数识别结果

\begin{tabular}{cccccc}
\hline \multirow{2}{*}{ 符号 } & \multicolumn{2}{c}{ 附加质量法 } & & \multicolumn{2}{c}{ 比例关系法 } \\
\cline { 2 - 3 } \cline { 5 - 6 } & 识别值 & 误差(\%) & & 识别值 & 误差(\%) \\
\hline$m_{\mathrm{b}}$ & 35997.9898 & -0.0558 & 36000.0326 & 0.0009 \\
$J_{\mathrm{b}}$ & 2299938.3395 & -0.0268 & 2285288.0891 & -6.3965 \\
$m_{\mathrm{t}}$ & 2099.9697 & -0.0144 & - & - \\
$J_{\mathrm{t}}$ & 2099.9786 & -0.0102 & 2100.0007 & 0.0003 \\
$k_{\mathrm{s}}$ & 259986.4419 & -0.0521 & 260000.4652 & 0.0018 \\
$c_{\mathrm{s}}$ & 19999.5032 & -0.0248 & 20000.0024 & 0.0001 \\
$k_{\mathrm{p}}$ & 599992.0640 & -0.0132 & 600000.0683 & 0.0001 \\
$c_{\mathrm{p}}$ & 29999.3238 & -0.0225 & 29999.9974 & -0.0001 \\
\hline
\end{tabular}

\section{表 3 模态空间理论物理参数识别结果}

\begin{tabular}{cccccc}
\hline \multirow{2}{*}{ 符号 } & \multicolumn{2}{c}{ 附加质量法 } & & \multicolumn{2}{c}{ 比例关系法 } \\
\cline { 2 - 3 } \cline { 5 - 6 } & 识别值 & 误差(\%) & & 识别值 & 误差(\%) \\
\hline$m_{\mathrm{b}}$ & 35999.7650 & -0.0065 & 35999.9951 & -0.0001 \\
$J_{\mathrm{b}}$ & 2298522.4742 & -0.6424 & 2285610.1216 & -6.2565 \\
$m_{\mathrm{t}}$ & 2100.0003 & 0.0001 & - & - \\
$J_{\mathrm{t}}$ & 2100.0002 & 0.0001 & 2099.9999 & $-1.05 \times 10^{-5}$ \\
$k_{\mathrm{s}}$ & 259998.6376 & -0.0052 & & 259999.9992 & $-3.02 \times 10^{-6}$ \\
$c_{\mathrm{s}}$ & 19999.9004 & -0.0050 & & 20000.0002 & $1.05 \times 10^{-5}$ \\
$k_{\mathrm{p}}$ & 600000.8140 & 0.0014 & & 600000.0042 & $7.05 \times 10^{-6}$ \\
$c_{\mathrm{p}}$ & 30000.0578 & 0.0019 & 30000.0001 & $4.45 \times 10^{-7}$ \\
\hline
\end{tabular}

\section{5 结论}

基于状态空间理论和模态空间理论, 分别提出 了比例关系法和附加质量法两种参数识别方法, 对 铁道车辆质量参数、惯量参数和悬挂参数同时识别 进行了探索性研究, 设计了用于参数识别的定向激 励测试工况, 并通过仿真试验对以上参数识别方法 的有效性进行了验证。得到以下主要结论。
(1) 采用附加质量法进行参数识别时, 附加质 量大小选取为车辆系统质量的 $3 \%$ 识别精度最高。

(2) 就车体质量参数、构架点头转动惯量参数 及悬挂参数而言, 基于模态空间理论的识别精度要 高于状态空间理论, 比例关系法的识别精度要高于 附加质量法。

(3) 就车体点头转动惯量参数而言, 附加质量 法的识别精度要高于比例关系法, 且基于状态空间 理论的附加质量法识别精度要高于基于模态空间理 论的附加质量法。

（4）若要识别构架质量参数, 只能采用附加质 量法, 此时, 基于模态空间理论的识别精度要高于 状态空间理论。

比例关系法和附加质量法在识别精度和测试实 施难度方面各有优缺点。附加质量法可在车辆物理 参数完全未知的条件下进行参数识别, 但需要将特 定的质量附加到车辆系统中, 存在一定的测试难 度。而比例关系法无需附加质量, 操作方便, 但需 要已知构架质量参数。因此, 在实际车辆参数识别 中, 可根据现场情况, 合理选择识别方法。后续作 者将进一步对包含构架和车体侧滚转动惯量的三 维模型的物理参数识别进行研究, 并进行车辆现场 测试与验证。

\section{参 考 文 献}

[1] 周劲松. 铁道车辆振动与控制 $[\mathrm{M}]$. 北京: 中国铁道出 版社, 2012 .

ZHOU Jinsong. Vibration and control of railway vehicles[M]. Beijing: China Railway Press, 2012.

[2] 张兆丰. 铁道客车动力学性能灵敏度分析及稳健设计 [D]. 长沙: 中南大学, 2012.

ZHANG Zhaofeng. Sensitivity analysis and robust design of dynamic performance of railway passenger vehicle[D]. Changsha: Central South University, 2012.

[3] SUAREZ B, FELEZ J, RODRIGUEZ J M P. Sensitivity analysis to assess the influence of the inertial properties of railway vehicle bodies on the vehicle's dynamic behaviour[J]. Vehicle System Dynamics, 2013, 51(2): 251-279.

[4] ZHOU J S, SHEN G, ZHANG H, et al. Application of modal parameters on ride quality improvement of railway vehicles[J]. Vehicle System Dynamics, 2008, 46(1): 629-641.

[5] GONG D, ZHOU J S, SUN W J. On the resonant vibration of a flexible railway car body and its 
suppression with a dynamic vibration absorber[J]. Journal of Vibration \& Control，2013， 19(5): 649-657.

[6] 高臣善. 铁道机车车辆质量测定方法 JIS E40111986[J]. 国外铁道车辆, 1989(5): 51-54.

GAO Chenshan. Method for measuring the quality of railway locomotives JIS E4011-1986[J]. Foreign Railway Vehicles, 1989(5): 51-54.

[7] 藤田科平, 高魁源. 机车车辆质量特性测定方法 [J]. 国 外铁道车辆, 1985(1): 12-18.

FUJITA K P, GAO Kuiyuan. Method for determining the quality characteristics of locomotive vehicle[J]. Foreign Railway Vehicles, 1985(1): 12-18.

[8] SONG K S, CHOI Y S. Field Measurement of the Center of Gravity and the Moment of Inertia of Railway Vehicles Using Vibration[J]. Transactions of the KSNVE, 23(10): 878-884.

[9] 刘友梅. 机车重心计算与测定方法[J]. 机车电传动, 2000(6): 1-3.

LIU Youmei. Calculation and determination method of $\mathrm{cg}$ of locomotive[J]. Locomotive Electric Drive, 2000(6): $1-3$.

[10] 王秀刚, 苏建, 曹晓宁, 等. 转向架三轴向转动惯量测 试方法[J]. 华南理工大学学报, 2012, 40(11): 71-77. WANG Xiugang, SU Jian, CAO Xiaoning, et al. Test method of three-axis moment of inertia of bogies[J]. Journal of South China University of Technology, 2012, 40(11): 71-77.

[11] 曹晓宁, 刘玉梅, 苏建, 等. 转向架质心高度的测定 $[\mathrm{J}]$. 吉林大学学报, 2013, 43(2): 329-333

CAO Xiaoning, LIU Yumei, SU Jian, et al. Determination of mass center height of bogie[J]. Journal of Jilin University, 2013, 43(2): 329-333.

[12] 周建烽, 王勇, 伊建辉. 轨道车辆车体动力学参数测量 试验台研制 $[\mathrm{J}]$. 铁道车辆, 2015，53(7): 38-40.

ZHOU Jianfeng, WANG Yong, YI Jianhui. Development of test bench for vehicle dynamics parameters of rail vehicles[J]. Railway Vehicles, 2015， 53(7): 38-40.

[13] 李雪海. 高速列车转向架悬挂参数测试方法研究 [D]. 长春: 吉林大学, 2011

LI Xuehai. Research on measuring methods of high-speed train bogie suspension parameters[D]. Changchun: Jilin
University, 2011.

[14] 张瑞芳, 刘峰, 黄强. 灰箱辨识在二系悬挂参数估计中 的应用 $[J]$. 铁道机车车辆, 2006, 26(4): 26-28.

ZHANG Ruifang, LIU Feng, HUANG Qiang. Application of grey box identification in estimation of secondary suspension parameters[J]. Railway Locomotive， 2006， 26(4): 26-28.

[15] 张卫华, 陈良麒, 黄丽湘. 车辆参数测定方法的研究 $[\mathrm{J}]$. 铁道车辆, 2000(12): 1-4.

ZHANG Weihua, CHEN Liangqi, HUANG Lixiang. Research on vehicle parameter determination method[J]. Railway Vehicles, 2000(12): 1-4.

[16] 陈建政, 张卫华, 陈良麒. 车体参数测定方法研究 $[\mathrm{J}]$. 西南交通大学学报, 2000, 35(2): 155-159.

CHEN Jianzheng, ZHANG Weihua, CHEN Liangqi. On measuring methods for parameters of car body[J]. Journal of Southwest Jiaotong University, 2000，35(2): 155-159.

[17] GOODWIN M J. Dynamics of railway vehicle systems[J]. Journal of Mechanical Working Technology, 1984, 14(2): 245-247.

[18] 刘豹, 唐万生. 现代控制理论[M]. 3 版. 北京: 机械工 业出版社, 2006.

LIU Bao, TANG Wansheng. Modern control theory[M]. 3rd ed. Beijing: China Machine Press, 2006.

[19] 曹树谦. 振动结构模态分析: 理论、实验与应用 $[\mathrm{M}]$. 天 津: 天津大学出版社, 2001.

CAO Shuqian. Modal analysis of vibration structure: theory, experiment and application[M]. Tianjin: Tianjin University Press, 2001.

[20] 同济大学应用数学系. 矩阵分析 $[\mathrm{M}]$. 上海: 同济大学 出版社, 2005 .

Department of Applied Mathematics, Tongji University. Matrix analysis[M]. Shanghai: Tongji University Press, 2005.

[21] IBRAHAM S R. A method for the direct identification of vibration parameter from the free responses[J]. Shock \& Vibration Bulletin, 1977, 47: 35-42.

作者简介: 陈江雪, 男, 1994 年出生, 博士研究生。主要研究方向为铁 道车辆动力学。

E-mail: chenjiangxue@tongji.edu.cn

周劲松(通信作者), 男, 1969 年出生, 博士, 教授, 博士研究生导师。 主要研究方向为铁道车辆动力学。

E-mail: jinsong.zhou@tongji.edu.cn 